\title{
ON THE DETECTABILITY OF STAR-PLANET INTERACTION
}

\author{
Brendan P. Miller ${ }^{1}$, Elena Gallo ${ }^{1}$, Jason T. Wright ${ }^{2,3}$, and Andrea K. Dupree ${ }^{4}$ \\ ${ }^{1}$ Department of Astronomy, University of Michigan, Ann Arbor, MI 48109, USA \\ ${ }^{2}$ Department of Astronomy and Astrophysics, The Pennsylvania State University, University Park, PA 16802, USA \\ ${ }^{3}$ Center for Exoplanets and Habitable Worlds, The Pennsylvania State University, University Park, PA 16802, USA \\ ${ }^{4}$ Harvard-Smithsonian Center for Astrophysics, Cambridge, MA 02138, USA \\ Received 2012 May 2; accepted 2012 May 31; published 2012 July 18
}

\begin{abstract}
Magnetic (or tidal) interactions between "hot Jupiters" and their host stars can potentially enhance chromospheric and coronal activity. An ideal test bed for investigating this effect is provided by the extreme WASP-18 system, which features a massive $(\sim 10$ times Jupiter) close-in ( $\lesssim 1$ day period) transiting planet orbiting a young F6 star. Optical and X-ray observations of WASP-18 were conducted in 2011 November. The high-resolution echelle spectrograph Magellan Inamori Kyocera Echelle was used on the $6.5 \mathrm{~m}$ Magellan Clay Telescope to obtain 13 spectra spanning planetary orbital phases of 0.7-1.4, while the X-ray Telescope on Swift provided contemporaneous monitoring with a stacked exposure of $\sim 50 \mathrm{ks}$. The cores of the $\mathrm{Ca} \mathrm{II} \mathrm{H}$ and $\mathrm{K}$ lines do not show significant variability over multiple orbits spanning $\sim 8$ days, in contrast to the expectation of phase-dependent chromospheric activity enhancements for efficient star-planet interaction. The star is also X-ray faint, with $\log L_{X}<27.6$, indicating that coronal activity is likewise low. The lack of detectable star-planet interaction in this extreme system requires that any such effect here must be transient, if indeed present. We demonstrate that searches for $\mathrm{Ca} \mathrm{II} \mathrm{H}$ and $\mathrm{K}$ variability can potentially mistake a stellar hotspot, if observed over a short segment of the rotation period, for planet-induced activity. Taken together, these results suggest that the utility of star-planet interaction as a robust method of estimating exoplanet magnetic field strengths may be limited.
\end{abstract}

Key words: planetary systems - stars: activity - stars: individual (WASP-18) - stars: magnetic field

Online-only material: color figures

\section{INTRODUCTION}

The possibility of magnetic star-planet interactions was initially explored by Cuntz et al. (2000; see also Rubenstein \& Schaefer 2000), and this and subsequent work has indicated that reconnection events could be an important effect in "hot Jupiter" systems, acting to produce enhancements ${ }^{5}$ in chromospheric and coronal activity corotating with the planet rather than with the stellar rotation. It has been conjectured that the energy available from reconnection events should scale with the product of the stellar and planetary magnetic field strengths, as well as the relative velocity of the magnetic field lines, and inversely with approximately the square of the orbital semimajor axis (Cuntz et al. 2000; Kashyap et al. 2008). Consequently, a calibrated relationship between the amplitude of star-planet interaction and stellar and orbital parameters could permit estimation of exoplanet magnetic field strengths. Throughout this work, we quantify interaction strength in otherwise similar systems as $M_{\mathrm{P}} / a^{2}$, where $a$ is the semimajor axis in $\mathrm{AU}$ and the planetary magnetic field strength is taken to scale with the planetary mass $M_{\mathrm{P}}$ (e.g., Arge et al. 1995; Stevens 2005); this is broadly consistent with the various proposed trends investigated (e.g., Kashyap et al. 2008; Shkolnik et al. 2008; Scharf 2010; Poppenhäger et al. 2010). Numerical studies suggest that star-planet interactions can potentially generate sufficient energy to be observable as, e.g., phase-variable core $\mathrm{Ca}$ II $\mathrm{H}$ and $\mathrm{K}$ or X-ray emission (probing chromospheric and coronal activity, respectively) in monitoring of individual hot Jupiter systems, or as a greater average level of activity in systems with more

\footnotetext{
5 Tidal star-planet interaction is also possible, but likely relevant only in very close systems, if any, due to the inverse-cube scaling with semimajor axis. Any tidal effect should be maximal at planetary transit.
}

massive or closer-in planets. For example, Lanza (2008) and Lanza et al. (2011) modeled chromospheric hot spots in several systems (offset from the subplanetary point by varying degrees) as arising from star-planet magnetic reconnection events, and Cohen et al. (2009, 2011) carried out three-dimensional magnetohydrodynamic simulations demonstrating that close-in giant planets can produce an increase in overall stellar activity and generate (non-persistent) coronal hot spots that rotate synchronously with the planet (albeit potentially shifted in phase); see also Pillitteri et al. (2010).

Observational evidence of magnetic star-planet interaction has now been presented for several individual systems. For example, Shkolnik et al. (2005) examined 10 stars (K1 to F7) known at the time to possess massive, close-in planets (median minimum planetary mass $M_{\mathrm{P}} \sin i=0.6 M_{\mathrm{Jup}}$, median orbital period $P=3.4$ days), in low-eccentricity orbits, and claimed evidence of star-planet interaction in HD 179949 and $v$ And (both F8) based on slight Ca II $\mathrm{H}$ and $\mathrm{K}$ emission variability ${ }^{6}$ synchronized with the planetary period (see also discussion in $\mathrm{Gu}$ et al. 2005), although this synchronization is apparently transient (Shkolnik et al. 2008). Chandra observations of HD 179949 showed variable X-ray emission with an apparent maximum near the phase associated with $\mathrm{Ca}$ II $\mathrm{H}$ and $\mathrm{K}$ enhancement (Saar et al. 2008). Fares et al. (2012) carried out spectropolarimetric observations of HD 179949, finding that the stellar magnetosphere is highly tilted (producing two maxima per rotation period) and that chromospheric activity is primarily linked to stellar rotation, although low-level fluctuations near the beat period could be planet induced (see also Gurdemir

\footnotetext{
6 The cores of the $\mathrm{Ca}$ II $\mathrm{H}$ and $\mathrm{K}$ lines are good indicators of chromospheric activity; residuals from $\mathrm{H} \alpha$ or the calcium infrared triplet are also useful for this purpose.
} 
et al. 2012). Later Chandra observations of $v$ And (as well as concurrent optical spectroscopy) did not find any indications of star-planet interaction (Poppenhäger et al. 2011). Interaction was claimed for $\tau$ Boo (F7) based primarily on photometric spot modeling, although the tidal locking of the star to the planet complicates interpretation (Walker et al. 2008; see also Shkolnik et al. 2008). HD 189733 (K0) twice showed an X-ray flare after secondary eclipse (Pillitteri et al. 2010, 2011) but chromospheric variability appears to be tied to stellar rotation (Shkolnik et al. 2008; Fares et al. 2010). Optical spectroscopy of several systems (including $v$ And and $\tau$ Boo) carried out by Lenz et al. (2011) did not find evidence of planet-induced chromospheric activity, but did identify potential interaction between the M dwarf HD 41004B (which has a binary, planetbearing $\mathrm{K}$ dwarf companion HD 41004A) and its brown-dwarf companion HD 41004Bb. As a counterexample, 51 Peg (G5) is one of several systems in which a sensitive search did not uncover evidence of star-planet interaction; its low $L_{X}$ and low $\log R_{\mathrm{HK}}^{\prime}$ suggest it may in fact be in an extended state of especially low activity, analogous to the Sun's Maunder minimum (Poppenhäger et al. 2009).

Several other studies have used large samples of planetbearing stars to test for significantly increased activity in hot Jupiter systems, statistically averaging over orbital phase. X-ray investigations of potential planet-induced enhancements in coronal activity have to date provided mixed results. This may be due to the difficulty of controlling for selection effects (more active stars, which tend to be brighter in X-rays, are disproportionately identified as planet bearing with high-mass, close-in planets, since those stellar properties limit detectability of low-mass, distant planets) or may be related to sampling a heterogeneous mix of late-type (typically F, G, K, and M) stellar classes. Kashyap et al. (2008) considered a large sample primarily drawn from ROSAT pointed and all-sky survey (RASS) observations (containing $\sim 30 \% / 70 \%$ X-ray detections/upper limits) and found that, after attempting to control for selection effects, stars with close-in planets $(a<$ $0.15 \mathrm{AU}$ ) were 1.3-4 times more X-ray luminous than stars with distant ( $a>1.5$ AU) planets. Similarly, Scharf (2010), using RASS data again with a high percentage of upper limits, did not find enhanced X-ray emission for close-in planets (note that their distant planets encompassed $a>0.15 \mathrm{AU}$ ) and estimated their $L_{\mathrm{X}}-M_{\mathrm{P}}$ correlation to be robust against observational bias. On the other hand, Poppenhäger et al. (2010) looked at a volumelimited $(d<30 \mathrm{pc})$ sample of stars observed with XMM-Newton or ROSAT and found no significant correlations of $L_{\mathrm{X}} / L_{\mathrm{bol}}$ with $M_{\mathrm{P}}$ or $a$; they explain the possible correlation of $L_{\mathrm{X}}$ with $M_{\mathrm{P}} / a$ as arising entirely from selection effects. Importantly, Poppenhäger \& Schmitt (2011) demonstrated that distance in shallow RASS data sets covaries with both $L_{\mathrm{X}}$ and $M_{\mathrm{P}}$ (indicating that deeper observations are essential to sidestep selection effects) and that apparent correlations with $L_{\mathrm{X}}$ vanish when $L_{\mathrm{X}} / L_{\text {bol }}$ is used instead (thereby controlling, to first order, for spectral class). Large-sample studies of potential planet-induced enhancements in chromospheric activity have also given mixed results; Canto Martins et al. (2011) find no obvious correlations between planetary mass or semimajor axis and the $\mathrm{Ca}$ II $\mathrm{H}$ and $\mathrm{K}$ activity indicator $\log R_{\mathrm{HK}}^{\prime}$, whereas Krejčová \& Budaj (2012) do find these variables to correlate but only within the subset of cooler host stars with $T_{\text {eff }}<5500 \mathrm{~K}$.

In this paper, we present a sensitive search for star-planet interaction in the extreme WASP-18 system. If star-planet interaction is to become a useful probe of exoplanet magnetic field strengths in hot Jupiter systems, additional convincing instances, beyond the handful suggested to date, must be identified. Observations of a strongly interacting system could constitute a contextual template to guide interpretation of results in more weakly interacting systems (including those already studied). Additionally, a robust measurement of star-planet interaction in an extreme high-mass, short-period system would supply productive leverage for uncovering and later quantifying scaling relations. Conversely, a failure to detect planet-induced stellar activity in an extreme system would severely constrain the practical relevance of star-planet interaction and could impact theoretical understanding and numerical modeling of this effect.

\subsection{WASP-18 in Context}

We identified WASP-18 as the potentially most strongly interacting system among currently discovered exoplanets, a distinction it retains as of 2012 April. We obtained a list of all confirmed planets with $M_{\mathrm{P}}>0.1 M_{\mathrm{J}}$ (i.e., planetary mass greater than $10 \%$ that of Jupiter), orbital period $P<$ 100 days, and $V<15$ from the Exoplanet Orbit Database ${ }^{7}$ (Wright et al. 2011). As may be seen in Figure 1, WASP-18b (Hellier et al. 2009; Southworth et al. 2009), with $M_{\mathrm{P}}=$ $10.4 M_{\mathrm{J}}, P=0.941$ days, and $a=0.020 \mathrm{AU}$, has the largest value of the interaction strength proxy $M_{\mathrm{P}} / a^{2}$ within this sample (and would also rank first for other plausible parameterizations of interaction strength). More specifically, after excluding brown dwarfs (with $M_{\mathrm{P}}>13 M_{\mathrm{Jup}}$ ), of the 551 entries in the Exoplanet Orbit Database, the $M_{\mathrm{P}} / a^{2} \simeq 25,000 M_{\text {Jup }} \mathrm{AU}^{-2}$ for WASP-18 is nearly 2.5 times greater than the next largest value (CoRoT-14b), and only five systems have $M_{\mathrm{P}} / a^{2}>$ $5000 M_{\text {Jup }} \mathrm{AU}^{-2}$. Because WASP-18 is a transiting system, the planetary properties are securely established by light curve and radial velocity measurements. The values of $M_{\mathrm{P}} / a^{2}$ are also labeled for several systems described in Section 1; for example, HD 179949 has $M_{\mathrm{P}} / a^{2} \simeq 500 M_{\mathrm{Jup}} \mathrm{AU}^{-2}$.

The stellar and planetary properties for the WASP-18 system are given in Table 1, as are those for previously identified star-planet interaction candidates HD 179949, $v$ And, and $\tau$ Boo (but recall $\tau$ Boo is tidally locked, with stellar and orbital periods of $\sim 3.2$ days, which may suppress magnetic interaction). The value of $M_{\mathrm{P}} / a^{2}$ for WASP-18b exceeds that for the other candidate systems by one to two orders of magnitude. Such extreme planetary systems are difficult to maintain, and WASP-18b itself is expected to have a short lifetime against infall from tidal drag (Hellier et al. 2009). The star WASP-18 has spectral type F6, effective temperature $T_{\text {eff }}=6400 \pm 100 \mathrm{~K}$, and stellar mass $M_{*}=1.22 \pm 0.03 M_{\odot}$ (values from the Exoplanet Orbit Database, from which original references may be obtained); conveniently, these are similar to the properties of HD 179949 $\left(\mathrm{F} 8,6170 \mathrm{~K}, 1.18 M_{\odot}\right), v$ And $\left(\mathrm{F} 8,6210 \mathrm{~K}, 1.31 M_{\odot}\right)$, and $\tau$ Boo $\left(\mathrm{F} 7,6390 \mathrm{~K}, 1.34 M_{\odot}\right)$. WASP-18 is slightly hotter than HD 179949 and $v$ And, and consequently likely possesses a slightly shallower outer convection zone. On the other hand, the stellar rotation period is shorter, at $\sim 5.6$ days, estimated from $v \sin i=11 \mathrm{~km} \mathrm{~s}^{-1}$; the orbit and stellar rotation are aligned $^{8}$ (Triaud et al. 2010). HD 179949 and $v$ And have rotational periods of 7.6 days (Fares et al. 2012) and $\sim 12$ days (Shkolnik et al. 2008), respectively. Further, WASP-18 appears

\footnotetext{
7 Available at http://www.exoplanets.org.

8 All four of these systems are near the $T_{\text {eff }} \sim 6250 \mathrm{~K}$ border above which hot Jupiter hosts tend to have high stellar obliquities (Winn et al. 2010), but the spin-orbit alignment in WASP-18 is more characteristic of cooler hot Jupiter hosts.
} 


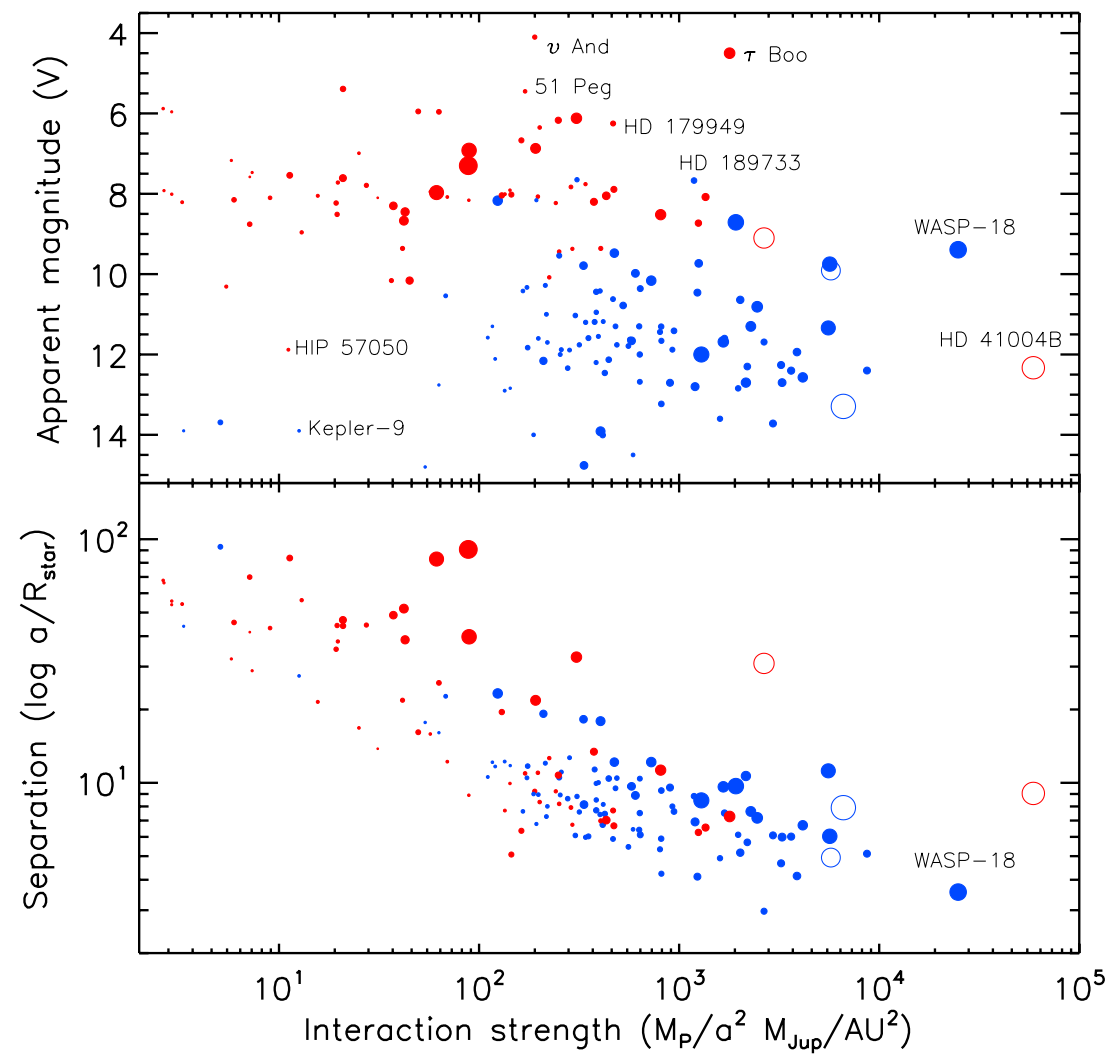

Figure 1. Distribution of star-planet interaction strength, assumed here to scale as $M_{\mathrm{P}} / a^{2}$, plotted vs. apparent $V$ magnitude (top) and star-planet separation in units of stellar radii (bottom). Note that the horizontal axis is logarithmic. Transiting planets are plotted in blue, and symbol size scales as $M_{\mathrm{P}}^{0.5}$. Brown dwarfs ( $>13 M_{\mathrm{J}}$ ) are plotted as open symbols. Radial velocity masses are lower limits, but establishing $\sin i$ would typically result in only modest rightward movement of those points. Several relevant or interesting systems are labeled (the $b$ planet designation is implicit).

(A color version of this figure is available in the online journal.)

Table 1

Candidates for Star-Planet Interaction

\begin{tabular}{|c|c|c|c|c|c|c|c|c|c|c|c|c|}
\hline \multirow[b]{2}{*}{ Name } & \multicolumn{4}{|c|}{ Star } & \multicolumn{3}{|c|}{ Planet } & \multicolumn{5}{|c|}{ Activity } \\
\hline & Type & V & $B-V$ & $\begin{array}{c}d \\
(\mathrm{pc})\end{array}$ & $\begin{array}{c}M_{\mathrm{P}} \\
\left(M_{\mathrm{J}}\right)\end{array}$ & $\begin{array}{c}P \\
\text { (days) }\end{array}$ & $\begin{array}{c}a \\
(\mathrm{AU})\end{array}$ & $\begin{array}{l}\text { Age } \\
(\mathrm{Gyr})\end{array}$ & $\begin{array}{c}M_{P} / a^{2} \\
\left(M_{\mathrm{J}} / \mathrm{AU}^{2}\right)\end{array}$ & $R_{\mathrm{HK}}^{\prime}$ & $\begin{array}{c}\log L_{\mathrm{X}} \\
\left(\mathrm{erg} \mathrm{s}^{-1}\right)\end{array}$ & $\log \left(\frac{L_{\mathrm{X}}}{L_{\mathrm{bol}}}\right)$ \\
\hline WASP-18 & F6 & 9.39 & 0.49 & 100 & 10.4 & 0.94 & 0.0201 & 0.6 & 25700 & $<-5.0^{\mathrm{a}}$ & $<27.6$ & $<-6.2$ \\
\hline$\tau$ Boo $^{b}$ & F7 & 4.50 & 0.51 & 15.62 & 4.1 & 3.31 & 0.0480 & 2.5 & 1780 & -4.73 & 28.8 & -5.3 \\
\hline HD 179949 & F8 & 6.25 & 0.55 & 27.5 & 0.95 & 3.09 & 0.0439 & 2.1 & 490 & -4.80 & 28.6 & -5.3 \\
\hline$v$ And & F8 & 4.10 & 0.54 & 13.49 & 0.69 & 4.62 & 0.0594 & 3.8 & 200 & -5.07 & 27.6 & -6.5 \\
\hline
\end{tabular}

Notes. Data are taken from the Exoplanet Orbit Database (http://www.exoplanets.org), except for planetary mass and system age which are from the Extrasolar Planets Encyclopedia (http://www.exoplanet.eu), and $\log L_{X}$ which is from this work, Poppenhaeger et al. (2012), Kashyap et al. (2008), and Poppenhäger et al. (2010) for WASP-18, $\tau$ Boo, HD 179949, and $v$ And, respectively; $\log \left(L_{\mathrm{X}} / L_{\mathrm{bol}}\right)$ is calculated.

${ }^{a}$ Estimated from our measured $\log L_{\mathrm{X}}<27.5 \mathrm{erg} \mathrm{s}^{-1}$ and the $R_{\mathrm{HK}}^{\prime}-\log \left(L_{\mathrm{X}} / L_{\mathrm{bol}}\right)$ correlation in Mamajek \& Hillenbrand (2008). See also footnote 15 .

$\mathrm{b}$ The potential strength of magnetic star-planet interaction in the $\tau$ Boo system is reduced due to the apparent tidal locking of the star to the planet; see Section 1.

to be a younger star, with an age of $\sim 500-700$ Myr (Hellier et al. 2009; Brown et al. 2011). The age-rotation-activity relation would then predict relatively greater intrinsic activity in WASP-18, apart from any planet-induced modulation or enhancement (but see Section 3.2 for caveats). Observationally, WASP-18 has other appealing features: the star is among the brighter transit-detected systems, and the short planetary period facilitates rapid accumulation of phase coverage across multiple orbits. Due to its extreme properties, WASP-18 has been studied at a range of frequencies; for example, Nymeyer et al. (2011) used Spitzer observations of secondary eclipse to infer that the planet has $T \simeq 3100 \mathrm{~K}$ with near-zero values for both albedo and day/night side energy redistribution. However, high-resolution, high signal-to-noise $\mathrm{Ca}$ II $\mathrm{H}$ and $\mathrm{K}$ spectroscopy, and sensitive
X-ray observations have not been published prior to the observations presented here.

\section{OBSERVATIONS}

\subsection{Data Acquisition and Reduction}

Optical spectroscopy was carried out with the $6.5 \mathrm{~m}$ Clay Telescope on 2011 November 6-7 and 10-13 using the Magellan Inamori Kyocera Echelle (MIKE), a high-throughput double echelle spectrograph. A $0^{\prime \prime} \cdot 7 \times 5^{\prime \prime}$ slit was used throughout, providing a resolution of $R \simeq 40,000$ near the Ca II H and $\mathrm{K}$ lines. A communication board failure prevented use of the blueside CCD on November 10 and bad weather limited observing on November 11. In total, 13 visits to WASP-18 were obtained, 


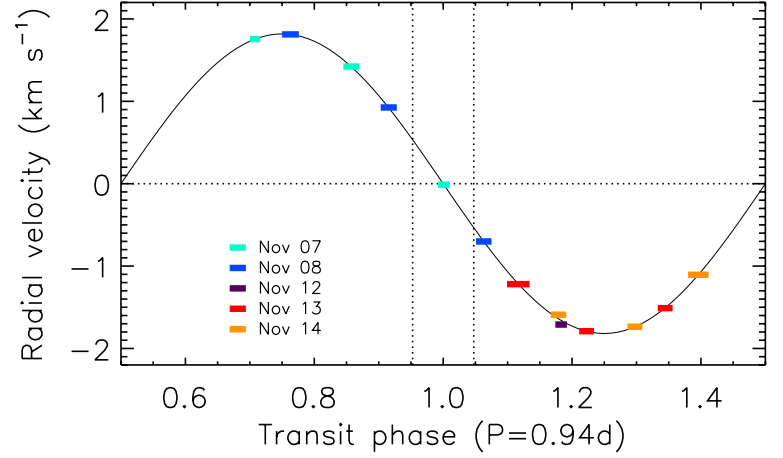

Figure 2. Phase coverage of the Magellan observations, plotted on the stellar radial velocity curve from the ephemeris of Hellier et al. (2009; error here $<0.001)$. Dates are UT 2011; phase coverage of the 0.94 day planetary orbit was accumulated over five nights. The November 12 and first November 14 points have slight vertical offsets for clarity. The vertical dotted lines mark planetary transit.

(A color version of this figure is available in the online journal.)

with combined exposure times of 20-40 minutes per visit. The planetary orbital phase coverage (Figure 2) spans $0.7-1.4$ and includes one visit at central planetary transit.

The spectra were reduced using the IDL MIKE Redux ${ }^{9}$ package. Briefly, milky flats (taken of $\delta$ Ori with the diffuser) were combined and normalized to correct pixel response variations; trace flats (from internal lamps) were combined for order and slit tracing; arc images (including one associated with each science exposure) were processed and used to derive two-dimensional wavelength maps, using a fifth-degree polynomial fit along the orders which was found to give generally structureless residuals; the slit profile was determined for each order; science exposures were processed (bias-subtracted from overscan and flat-corrected), the object was traced and the sky subtracted, and the object flux was optimally extracted. Flux is retained in relative units (i.e., no spectrophotometric standard star calibration was conducted) as we are interested in normalized spectra. Automated cosmic-ray correction was not performed due to concern about potential introduction of spurious variability; two science frames with an obvious cosmic ray located near the two-dimensional center of the CaII $\mathrm{K}$ line were discarded.

Contemporaneous monitoring was obtained with the Swift satellite from November 1-17 (Target ID 32149), notably with the X-ray Telescope (XRT) through 58 snapshots of 500-1500 s each. The XRT observations were reduced using HEASARC version 6.11.1. An exposure map was generated for each sequence, including a vignetting correction and with timedependent instrument maps used whenever attitude variations exceeded 2".4. Images were extracted in both the full $(0.3-10 \mathrm{keV})$ and soft $(0.3-2 \mathrm{keV})$ bands. The stacked XRT effective exposure from all sequences at the position of WASP18 reached $\sim 50 \mathrm{ks}$. The UV/Optical Telescope was also used to obtain both photometry (with the $U, U V W 1, U V M 2$, and $U V W 2$ filters) and UV grism spectroscopy, with coverage of the $\mathrm{Mg}$ II $\mathrm{h}$ and $\mathrm{k}$ lines which are also chromospheric activity indicators (Buccino \& Mauas 2008).

\subsection{Analysis and Results}

We checked for phase-dependent variability, as from onesided-enhanced chromospheric activity, within the cores of the $\mathrm{Ca}$ II $\mathrm{H}$ and $\mathrm{K}$ lines, focusing primarily upon the latter. The

\footnotetext{
9 http://web.mit.edu/ burles/www/MIKE/
}

Ca II $\mathrm{K}$ line and nearby stellar continuum were isolated through selecting wavelengths spanning 3920-3950 A, which included contributions from two orders. For each visit, the flux from individual exposures was summed, and the wavelength scale was adjusted through subtraction of a center-of-mass velocity of $3.1961 \mathrm{~km} \mathrm{~s}^{-1}$ and a stellar reflex velocity with an amplitude of $1.8183 \mathrm{~km} \mathrm{~s}^{-1}$, using the values and ephemeris from Hellier et al. (2009). Continuum normalization near the Ca II H and $\mathrm{K}$ regions is difficult because the absorption lines are quite broad relative to the blaze function; there are a large number of nearby metal lines, and the Wien tail is falling off steeply. We used a similar normalization method to that conducted and detailed by Shkolnik et al. (2005, 2008), removing a slight linear trend over $\simeq 7 \AA$ centered near the line cores, such that unity represents approximately one-third of the local stellar continuum. The normalized $\mathrm{Ca}$ II $\mathrm{H}$ and $\mathrm{K}$ spectra for all 13 visits are plotted in Figure 3 (top).

Residuals from median-normalized $\mathrm{Ca}$ II $\mathrm{H}$ and $\mathrm{K}$ spectra were calculated for each visit, and then stacked (weighted by exposure times corrected for seeing) within orbital phase bins of $0.70-0.93,0.99-1.19$, and $1.21-1.41$; these bins then contain spectra from four, five, and four visits, respectively. The residuals were boxcar smoothed with a width of $0.4 \AA$ and then any remaining slight linear trend was subtracted to avoid influencing comparison of the line core. There is no obvious difference in the residual spectra within the three separate phase bins, to an amplitude of 0.003 of the normalized continuum (Figure 3, bottom). Integrating the normalized flux over the $1 \AA$ line cores within phase bins of $0.70-0.93,0.99-1.19$, and $1.21-1.41$ gives nearly identical values: $0.252 \pm 0.001,0.251$ \pm 0.002 , and $0.253 \pm 0.003$ for $\mathrm{K}$ and $0.225 \pm 0.001,0.227$ \pm 0.001 , and $0.228 \pm 0.003$ for $\mathrm{H}$, where the $1 \sigma$ uncertainties have been estimated as the standard deviation of the component flux measurements within each phase bin. The $3 \sigma$ limit on the relative flux variability is $<3.2 \%$ and $<3.4 \%$ for the $\mathrm{K}$ and $\mathrm{H}$ line cores, respectively. The apparent marginal tendency for the $1.21-1.41 \mathrm{~K}$ bin to show relatively more red-side emission is within the noise level ${ }^{10}$ but in any case this slight profile variation is not present in $\mathrm{H}$, is lopsided rather than symmetric about the core, and lags rather than leads the subplanetary point; these effects are inconsistent with planetary magnetic interaction.

The XRT observations were initially planned for similar phase-resolved analysis, but the star proved to be unexpectedly X-ray faint (see also Section 3.2) and so all sequences were combined for an effective exposure of $\sim 50 \mathrm{ks}$. WASP-18 is not detected by XRT in the stacked exposure, in either the soft (Figure 4) or the full bands. Specifically, within an aperture of $20^{\prime \prime}$ centered on the SIMBAD optical position of WASP-18, there are 3.8 counts in the $0.3-2 \mathrm{keV}$ band, where the expected background is 3.7 counts. The $95 \%$ upper limit on the source counts within $20^{\prime \prime}$ is $<5.9$ net counts (from Kraft et al. 1991), corresponding to $<8.1$ total net counts after accounting for the XRT point-spread function (Moretti et al. 2005). For a coronal model with $k T=1 \mathrm{keV}$ and solar abundances, the unabsorbed $0.3-2 \mathrm{keV} \mathrm{X}$-ray flux is $<3.3 \times 10^{-15} \mathrm{erg} \mathrm{s}^{-1}$, calculated using the Portable Interactive Multi-Mission Simulator ${ }^{11}$ for a plasma/APEC model presuming an intervening column of $N_{\mathrm{H}}=10^{18}-10^{19} \mathrm{~cm}^{-2}$; other plausible models give similar

\footnotetext{
10 This may be verified through comparison to the residuals near photospheric absorption features, which should not show any variability phased with the planetary orbit.

11 http://cxc.harvard.edu/toolkit/pimms.jsp
} 


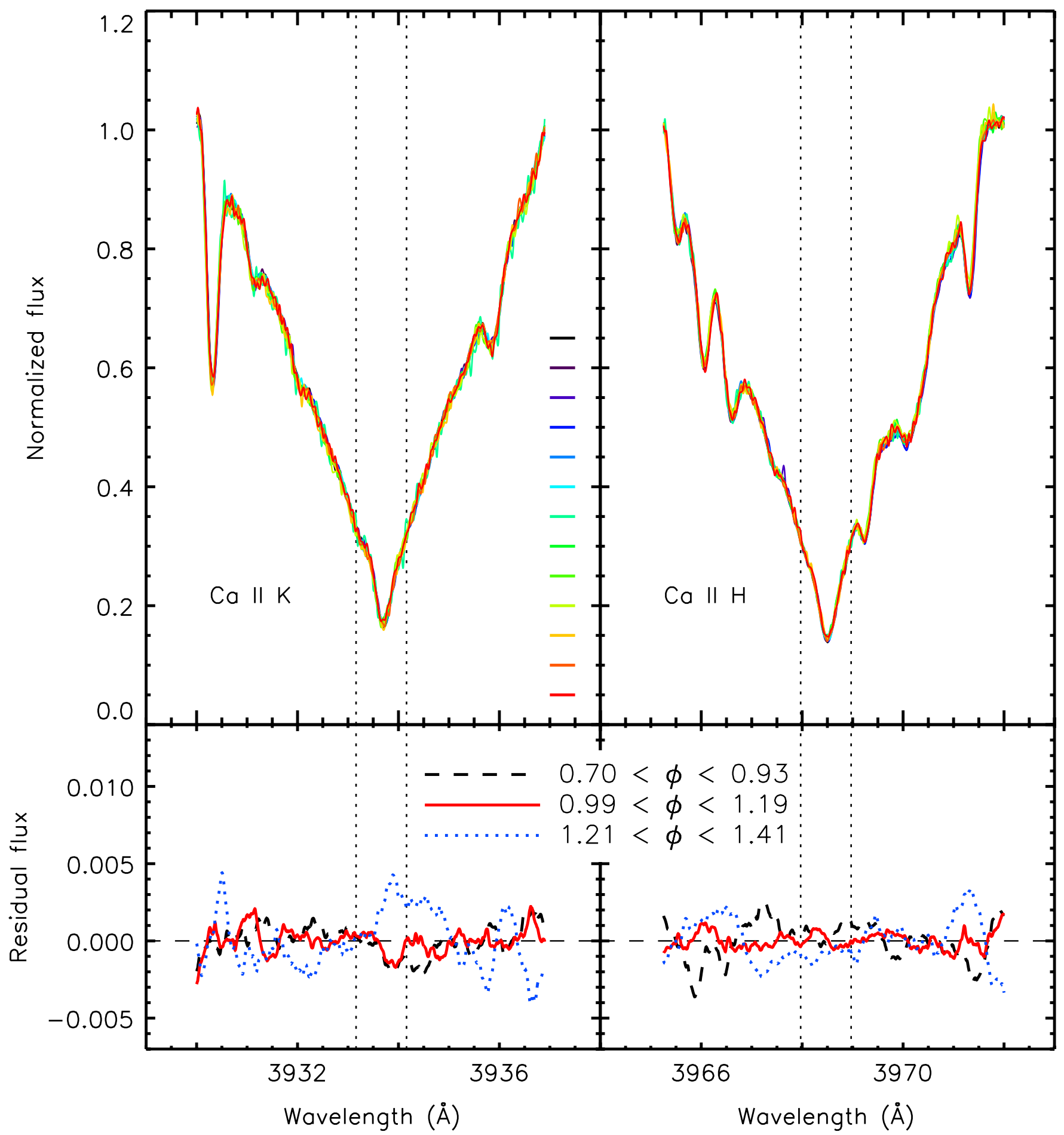

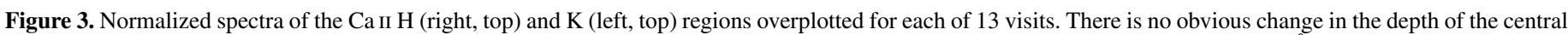

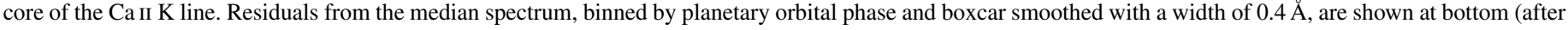
removal of slight linear trends). The vertical dotted lines mark the $\mathrm{Ca} \mathrm{II} \mathrm{H}$ and $\mathrm{K}$ cores.

(A color version of this figure is available in the online journal.)

results to within $\lesssim 30 \%$ (or $0.1 \mathrm{dex}$ ). At the Hipparcos distance to WASP-18 of $100 \pm 10.6 \mathrm{pc}$, this corresponds to a limiting $\mathrm{X}$-ray luminosity of $\log L_{\mathrm{X}}<27.6 \mathrm{erg} \mathrm{s}^{-1}(0.3-2 \mathrm{keV})$.

\section{DISCUSSION}

We compare the observed persistent low activity in WASP-18 to expectations for planet-induced variability, as well as to the intrinsic properties of similar stars, and additionally explore the general possibility of stellar hotspots acting to mimic star-planet interaction.

\subsection{Expected Variability in WASP-18}

Past work would seem to suggest that the degree of planetinduced $\mathrm{Ca}$ II $\mathrm{H}$ and $\mathrm{K}$ variability expected for WASP-18 could be substantial. For example, Shkolnik et al. $(2005,2008)$ report "on/off" Ca II H and K variability phased with the planetary 


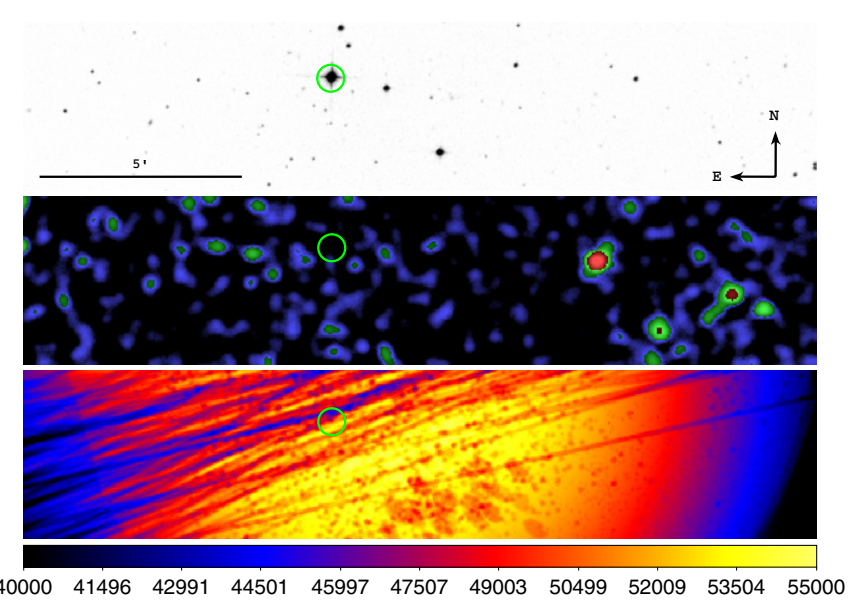

Figure 4. Top: Digital Sky Survey image of WASP 18. Middle: Swift XRT $0.3-2 \mathrm{keV}$ image of WASP 18 region, Gaussian smoothed with a kernel of 7 pixels and shown with logarithmic scaling. The green circle has a radius of $20^{\prime \prime}$ and is centered on the SIMBAD position of WASP 18. Bottom: XRT exposure map; the color bar gives the effective exposure in seconds.

(A color version of this figure is available in the online journal.)

orbital period in HD 179949 and $v$ And (see also Poppenhäger et al. 2011), which have stellar types of F8 and do not show obvious core emission within the deep photospheric absorption (similar to WASP-18). The amplitude of residual flux associated by Shkolnik et al. (2008, Figure 3; Shkolnik et al. 2005, Figure 6) with star-planet activity in HD 179949 ( $v$ And) is $\simeq 0.017$ $(\simeq 0.008) .{ }^{12}$ The value of $M_{\mathrm{P}} / a^{2}$ for WASP-18 could suggest an effect 50 (130) times greater than for HD 179949 ( $v$ And) ${ }^{13}$ In contrast to such predictions, the level of phase-binned variability observed for WASP-18 is $\lesssim 0.003$ (Figure 3; Section 2.2), below the levels detected in HD 179949 and $v$ And.

The degree of X-ray variability expected in WASP-18 is more difficult to estimate, as only a handful of X-ray-brightening events have been attributed to potential star-planet interaction. For example, HD 179949 displayed an increase in $\mathrm{X}$-ray emission by $\sim 30 \%$ near the phase associated with the $\mathrm{Ca}$ II H and $\mathrm{K}$ enhancement (Saar et al. 2008); with a stellar X-ray luminosity of $\log L_{\mathrm{X}}=28.6 \mathrm{erg} \mathrm{s}^{-1}$ (Kashyap et al. 2008), this is an increase of $\log L_{X}=28.1 \mathrm{erg} \mathrm{s}^{-1}$. HD 189733 showed two X-ray flares near phase 0.53 (i.e., shortly after occultation), peaking at twice the baseline count rate (Pillitteri et al. 2011); with a stellar X-ray luminosity of $\log L_{X}=28.4 \mathrm{erg} \mathrm{s}^{-1}$ (Kashyap et al. 2008), this is an increase of $\log L_{X}=28.4 \mathrm{erg} \mathrm{s}^{-1}$. Cohen et al. (2011) used their MHD modeling of the HD 189733 system to estimate that the energy available from magnetic reconnection to accelerate particles into the stellar corona is $\sim 10^{28} \mathrm{erg} \mathrm{s}^{-1}$ (after applying conservative efficiency assumptions). From these examples, WASP18 might be expected, taking into account its exceptional value of $M_{\mathrm{P}} / a^{2}$, to possess interaction energy available for coronal activity enhancement sufficient to generate absolute increases in its X-ray luminosity by several times $10^{28} \mathrm{erg} \mathrm{s}^{-1}$. However, the observed XRT upper limit of $\log L_{\mathrm{X}}<27.6 \mathrm{erg} \mathrm{s}^{-1}$ indicates that the X-ray luminosity did not achieve this level for any more than $\lesssim 10 \%$ of the Swift coverage. Without even a stacked X-ray

\footnotetext{
12 The ratio of residuals for HD 179949 and $v$ And is $\sim 2$, while the ratio of $M_{\mathrm{P}} / a^{2}$ is $\sim 2.5$. However, given the dissimilarity of other parameters, such as intrinsic stellar activity, and the apparent variability of any star-planet interaction in these systems, we do not consider this necessarily informative. 13 While less physically motivated, alternative scalings with either separately $1 / a^{2}$ or $M_{\mathrm{P}}$ would still predict a substantially greater effect for WASP-18, by a factor of 4.8 (8.7) or 11 (15) compared to HD 179949 ( $v$ And).
}

detection it is not possible to evaluate the relative amplitude of phase-dependent X-ray variability, if any, in WASP-18.

The scaling of star-planet interaction strength with $M_{\mathrm{P}} / a^{2}$ that we (and others; Section 1) have adopted approximately holds for otherwise similar systems. We briefly consider the impact upon the energy available for star-planet interaction due to potentially differing planetary or stellar magnetic field strengths in WASP-18 compared to HD 179949 and $v$ And. The planetary magnetic field strength depends most relevantly upon $M_{\mathrm{P}}$ but also scales inversely with the planetary rotational period. For tidally locked planets, as all these are believed to be (out to $\sim 0.15 \mathrm{AU}$; Bodenheimer et al. 2001), the planetary rotation period is identical to the orbital period, and both are substantially shorter for WASP-18 than for HD 179949 or $v$ And. This suggests (Arge et al. 1995; Sánchez-Lavega 2004; Stevens 2005) an increase in baseline planetary magnetic field strength by a factor of a few, which would enhance any star-planet interaction. The relative velocity between magnetic field lines, which is governed by the difference between the planetary and stellar rotation rates, is $v_{\text {rel }}=K\left(R_{*} / a\right)-v_{\text {rot }}$ for tidally locked planets, where $K$ is the orbital velocity and $v_{\text {rot }}$ is the equatorial stellar rotational velocity (Cuntz et al. 2000). This would suggest a further increase in interaction strength by a factor of a few. On the other hand, the interaction strength approximately scales with the stellar magnetic field, $B_{*}$ (Cuntz et al. 2000; Kashyap et al. 2008; see Lanza 2009 and Scharf 2010 for slightly different formulations), which appears to be substantially weaker in WASP-18 than in at least HD 179949 and perhaps also $v$ And. This is evident by the X-ray nondetection of WASP-18, as $B_{*}$ is observed to depend approximately linearly upon $L_{X}$ (Pevtsov et al. 2003). That $\log \left(L_{X} / L_{\text {bol }}\right)$ is at least one order of magnitude lower in WASP-18 than HD 179949 possibly offsets any gains in interaction strength due to faster planetary rotation, although the difference in inferred stellar magnetic field strengths is less between WASP-18 and $v$ And. However, since the observed amplitude of $\mathrm{Ca}$ II $\mathrm{H}$ and $\mathrm{K}$ variability in WASP-18 is already $\gtrsim 5$ times lower than that suggested to arise from star-planet interaction in HD $179949, B_{*}$ would need to be $\gtrsim 50$ times lower in WASP-18 than in HD 179949 to explain our nondetection for a similar interaction efficiency, which seems implausible given their similar stellar spectral types.

The lack of evidence for star-planet interaction in WASP18 is unexpected given that we selected it as the system with the greatest predicted interaction strength (based on planetary mass and semimajor axis) and given the broad similarity in stellar properties between WASP-18 and other systems (notably HD 179949 and $v$ And) for which star-planet interaction has been claimed. Star-planet interaction in WASP-18 would seem to be at best highly transient as it was not demonstrably present during our observations. For reference, $\mathrm{Ca}$ II $\mathrm{H}$ and $\mathrm{K}$ variability has been described as phased with the planetary orbit in $\lesssim 50 \%$ of the epochs at which HD 179949 and $v$ And have been observed. Further, the timescale for a magnetic reconnection event in the simulations of Cohen et al. (2011) is short, $\sim 0.25$ orbits. On the other hand, our spectroscopic coverage of WASP-18 extends over eight complete planetary orbits, and the X-ray observations cover many more (albeit at a lower sensitivity per orbit). To the extent that star-planet interactions are transient, they should intuitively occur with greater frequency in extreme systems such as WASP-18, which contrasts (if not definitively) with our findings. It might be alternatively suggested that, if our observations did not simply happen to take place within a period of relative quiescence, the efficiency of star-planet interaction 
for WASP-18 could be lower than has been identified in the past for less extreme systems. However, we emphasize again that WASP-18 is not notably distinctive in terms of stellar properties from HD 179949 and $v$ And. (Below, we explore the possibility that the particularly massive and close-in planet in WASP-18 actually acts to suppress stellar activity.)

Regardless of its underlying cause, this lack of observed variability in WASP-18 demonstrates that even extreme systems, arguably the best candidates to display planet-induced activity enhancements, challenge prevailing ideas concerning star-planet interaction. The appealing prospect of calibrating star-planet interactions to estimate exoplanet magnetic field strengths would currently appear to require additional unambiguous evidence of such interactions occurring.

\subsection{Expected Intrinsic Activity in WASP-18}

We next consider the overall baseline level of chromospheric and coronal activity in WASP-18, to provide context for the nondetection of planet-induced variability. WASP-18 shows atypically low $\mathrm{Ca}$ II $\mathrm{H}$ and $\mathrm{K}$ core emission for its spectral class and inferred age (contrast with, e.g., HD 111456; Freire Ferrero et al. 2004). When stellar age is calculated from Ca II activity, main-sequence $\mathrm{F}, \mathrm{G}, \mathrm{K}$, or $\mathrm{M}$ stars with inferred ages of 340-740 Myr (i.e., similar to the age of WASP-18, which is not based on Ca II activity ${ }^{14}$ ) are chromospherically "active" (following the definition of Henry et al. 1996), with characteristic values of $-4.5<R_{\mathrm{HK}}^{\prime}<-4.4$ (from data in Wright et al. 2004). While stars with $0.48<B-V<0.52$, as for WASP-18, display a wide range of activity, they are significantly less likely to have $R_{\mathrm{HK}}^{\prime}<-5.0(19 \%$, versus $37 \%$ for F, G, K, and M stars; from data in Wright et al. 2004). However, $R_{\mathrm{HK}}^{\prime}$ in WASP-18 is low; if estimated indirectly based on the $R_{\mathrm{HK}}^{\prime}-\log \left(L_{\mathrm{X}} / L_{\text {bol }}\right)$ correlation presented in Mamajek $\&$ Hillenbrand (2008), the measured X-ray limit for WASP-18 suggests $R_{\mathrm{HK}}^{\prime} \lesssim-5.0 .^{15}$

The X-ray luminosity for WASP-18 is also lower than predicted. Nine nearby stars of spectral class F5V-F7V (i.e., matched to WASP-18) in a volume-limited ROSAT sample have $\mathrm{X}$-ray luminosities of $27.5 \mathrm{erg} \mathrm{s}^{-1}<\log L_{\mathrm{X}}<29.0 \mathrm{erg} \mathrm{s}^{-1}$, with mean and median values of $\log L_{\mathrm{X}} \simeq 28.3 \mathrm{erg} \mathrm{s}^{-1}$ (from data in Schmitt \& Liefke 2004). However, WASP-18 is younger than typical main-sequence stars and should consequently be more X-ray luminous. While we are not aware of a specific comprehensive study of young $\mathrm{F}$ stars, in general the X-ray luminosity of F5+ stars modestly exceeds that of $G$ stars (Schmitt 1997), and Hyades $G$ stars (hence of a similar age to WASP-18) have mean $\log L_{\mathrm{X}} \simeq 28.8 \mathrm{erg} \mathrm{s}^{-1}$ (Stelzer \& Neuhäuser 2001; Preibisch \& Feigelson 2005). Consequently, the XRT-derived limit of $\log L_{\mathrm{X}} \lesssim 27.6 \mathrm{erg} \mathrm{s}^{-1}$ marks WASP-18 as notably X-ray faint relative to comparable stars, with a $\log \left(L_{\mathrm{X}} / L_{\text {bol }}\right) \lesssim-6.2$, similar to that of the Sun.

In summary, not only is there no apparent enhancement in chromospheric or coronal activity in WASP-18 that might be linked to interaction with the planet, but rather, the above results, taken together, suggest that WASP-18 is unusually quiet for a young F6 star. It is possible that the stellar age is incorrect; the $1 \sigma$ error bars on the measurement given by Hellier et al.

\footnotetext{
14 The age of WASP-18 is estimated as $630_{-530}^{+950}$ Myr by Hellier et al. (2009) based on stellar isochrones, and as $579_{-250}^{+305}$ Myr by Brown et al. (2011) based on tidal interaction modeling.

15 A recent high signal-to-noise Keck/HIRES spectrum of WASP-18, calibrated as described in Wright et al. (2004), gives $R_{\mathrm{HK}}^{\prime}=-5.15(\mathrm{H}$. Isaacson 2012, private communication).
}
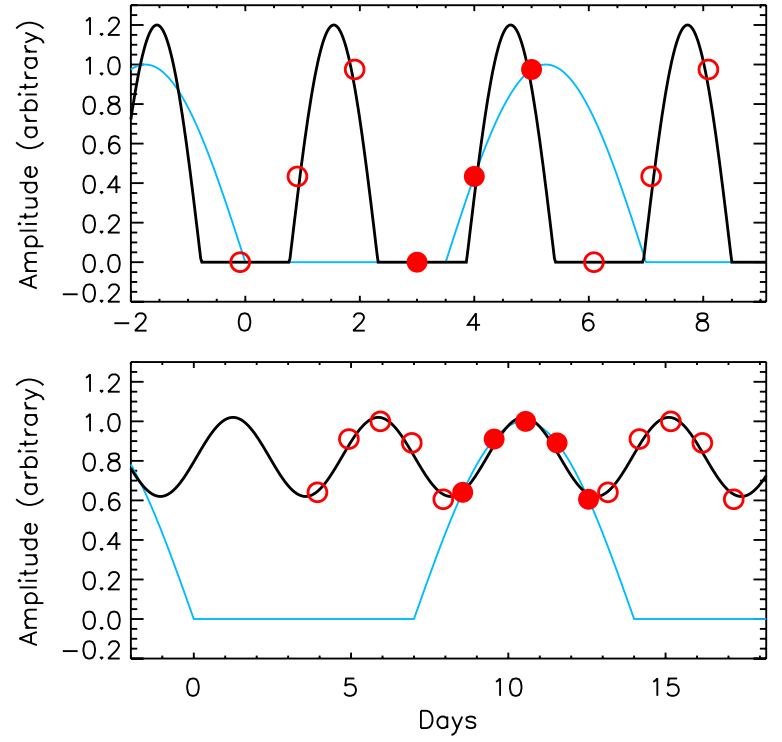

Figure 5. Examples of how a hotspot rotating with the star (blue line) could potentially mimic various models of star-planet interaction (black lines) over short observing runs (filled circles). The stellar hotspot phased with the planetary orbit is shown with open circles. See Section 3.3 for discussion.

(A color version of this figure is available in the online journal.)

(2009) encompass $\gtrsim 1$ Gyr, and the Brown et al. (2011) estimate depends upon complicated planet-star interactions. If the planet has acted to spin up the star (recall the orbital plane and the stellar rotation axis are aligned), the current relatively rapid stellar rotation rate would not be reflective of true age. Stellar age has proven difficult to determine in some other hot Jupiter systems; for example, Schröter et al. (2011) find that CoRoT-2a is X-ray bright and young but a late- $\mathrm{K}$ companion is X-ray undetected, inconsistent with the inferred system age, and a similar situation may apply for HD 189733 (Pillitteri et al. 2011). If WASP-18 were two to three times older than current estimates, the intrinsic activity would still be somewhat low but to a much less unusual degree. If the age is indeed correct, it is natural to consider whether the extremely massive and close-in planet could act to suppress, rather than enhance, stellar activity. The tidal force exerted by WASP-18 upon its parent star is much greater than is typical even for hot Jupiter systems, and in fact Arras et al. (2012) note that the small but apparently non-zero eccentricity indicated by radial velocity data is likely due to tidal fluid motion on the star. Mid-type F stars have shallow outer convective zones; perhaps the tidal pull is sufficient to repress dynamo activity in WASP-18 or comparably extreme systems (of which none are, however, currently known). This possibility could be assessed through magnetohydrodynamic simulations.

\subsection{An Additional Challenge to Observations of Star-Planet Interaction}

Here, we explore the possibility that a hotspot rotating upon the stellar surface can potentially mimic a shorter-period signature of star-planet interaction if only observed over a small fraction of the stellar rotation. Figure 5 shows simulated examples for HD 179949 and $v$ And, which have stellar/planetary periods of 7/3.09 and 14/4.618 days, respectively (Shkolnik et al. 2008). The half and full sinusoid models for planet-induced activity chosen for these examples are similar to those applied by Shkolnik et al. $(2005,2008)$ to these systems, but here for an edge-on inclination and with arbitrary 
normalizations. (By construction the stellar hotspot amplitude is taken as unity, and the relative amplitude of the interaction model is then greater for HD 179949 than for $v$ And, also qualitatively similar to the published models.) The probability of the stellar and planetary phases co-aligning to mimic star-planet interaction for these particular models is not very large (e.g., $\simeq 3 \%$ of 10,000 random phase offsets for each example yield total squared residuals less than 0.1 ), but with additional freedom to adjust the relative amplitudes, or to consider additional parameters (such as spot latitude or system inclination, within a limited range) or to choose from alternative model functional forms, or with a greater tolerance for (apparent) outliers, this effect could potentially present a significant source of contamination within a large sample of tested stars. For the particular cases of HD 179949 and $v$ And, it must be emphasized that several observing runs were conducted, and in some (but not all) of those runs variability of similar amplitude similarly phased with the planetary orbit was observed (Shkolnik et al. 2005, 2008; Poppenhäger et al. 2011), which clearly decreases the probability of mistaking a stellar hotspot for one corotating with the planet.

We note that, in general, searches for star-planet interaction based on Ca II core variability can guard against this type of false positive at a given epoch through obtaining coverage over at least one complete stellar rotation and over multiple planetary orbits (as was done with our coverage of WASP-18). If searches over multiple epochs find some instances in which core variability phases with the stellar rotation and others in which it apparently matches the orbital period, a comparison of the amplitudes can also check whether the observed activity is likely to arise from distinct (intrinsic versus planet-induced) sources. It would be odd if the activity in the "off" state (phased with stellar rotation rather than orbital period) had a similar amplitude to that seen in the "on" state, during which there is no obvious physical reason that the star should otherwise go silent. It is not clear to us that the current data for HD 179949 and $v$ And can definitively pass this test, but see, e.g., Fares et al. (2012). In any case, the conclusion is that complete and extended coverage, preferably across multiple epochs and multiple orbits per epoch, is essential for high-confidence detections of star-planet interaction.

\section{CONCLUSIONS}

We have carried out a sensitive search for planet-induced stellar activity within the extreme WASP-18 system, selected as an ideal test bed for investigating potential magnetic (or tidal) interactions between "hot Jupiters" and their host stars. Highresolution spectroscopy of the $\mathrm{Ca} \mathrm{II} \mathrm{H}$ and $\mathrm{K}$ lines was conducted with the $6.5 \mathrm{~m}$ Magellan Clay Telescope and contemporaneous $\mathrm{X}$-ray monitoring was obtained with the Swift satellite. Our primary results are the following.

1. The $\mathrm{Ca}$ II $\mathrm{H}$ and $\mathrm{K}$ cores do not show significant variability over $\sim 8$ days. Stacking residual spectra from 13 visits into phase bins of $0.70-0.93,0.99-1.19$, and $1.21-1.41$ does not show any significant structural changes that could be attributed to planetary influence.

2. WASP-18 is not detected in a stacked $50 \mathrm{ks}$ XRT exposure, constraining the X-ray luminosity to be unusually low for a young F6 star, with $\log L_{\mathrm{X}}<27.6$.

3 . The lack of observed variability phased with the planetary orbit suggests that any magnetic star-planet interaction in WASP-18 must be transient, if present at all.
4. The low level of chromospheric and coronal activity is consistent with an intrinsically weak magnetic field on WASP-18, perhaps indicating that the inferred young stellar age is not reliable, or alternatively potentially related to particularly strong planetary tidal effects.

5. It is demonstrated that a stellar hotspot can potentially mimic star-planet interaction, for observations truncated to a short segment of the rotation period.

6. Current ideas concerning star-planet interaction do not appear to be supported by the above results; therefore, it may be optimistic at present to conceive of star-planet interaction as a robust estimator of exoplanet magnetic field strengths.

Further high-quality Ca II $\mathrm{H}$ and $\mathrm{K}$ spectroscopy (as in Shkolnik et al. 2005, 2008) of previously monitored and newly discovered systems would refine understanding of the observational signatures of star-planet interaction. In addition, we are currently carrying out a Chandra survey of solar analogs to check whether stars with close-in planets are systematically enhanced in X-ray luminosity, and this experiment has been designed to sidestep many of the selection biases that necessarily challenged previous large-sample X-ray studies. That investigation will help further clarify whether the difficulties in establishing observational evidence of star-planet interaction in WASP-18 are anomalous or typical.

We thank Mario Mateo for essential assistance with the Magellan observing run, Niel Gehrels and the Swift Science Team for approving and carrying out our Target of Opportunity observations of WASP-18, and an anonymous referee for helpful comments. This paper includes data gathered with the 6.5 meter Magellan Telescopes located at Las Campanas Observatory, Chile. This research has made use of the Exoplanet Orbit Database and the Exoplanet Data Explorer at exoplanets.org. The Center for Exoplanets and Habitable Worlds is supported by the Pennsylvania State University, the Eberly College of Science, and the Pennsylvania Space Grant Consortium.

\section{REFERENCES}

Arge, C. N., Mullan, D. J., \& Dolginov, A. Z. 1995, ApJ, 443, 795

Arras, P., Burkart, J., Quataert, E., \& Weinberg, N. N. 2012, MNRAS, 422, 1761 Bodenheimer, P., Lin, D. N. C., \& Mardling, R. A. 2001, ApJ, 548, 466

Brown, D. J. A., Collier Cameron, A., Hall, C., Hebb, L., \& Smalley, B. 2011, MNRAS, 415, 605

Buccino, A. P., \& Mauas, P. J. D. 2008, A\&A, 483, 903

Canto Martins, B. L., Das Chagas, M. L., Alves, S., et al. 2011, A\&A, 530, A73 Cohen, O., Drake, J. J., Kashyap, V. L., et al. 2009, ApJ, 704, L85

Cohen, O., Kashyap, V. L., Drake, J. J., et al. 2011, ApJ, 733, 67

Cuntz, M., Saar, S. H., \& Musielak, Z. E. 2000, ApJ, 533, L151

Fares, R., Donati, J.-F., Moutou, C., et al. 2010, MNRAS, 406, 409

Fares, R., Donati, J.-F., Moutou, C., et al. 2012, MNRAS, 423, 1006

Freire Ferrero, R., Frasca, A., Marilli, E., \& Catalano, S. 2004, A\&A, 413, 657 Gu, P.-G., Shkolnik, E., Li, S.-L., \& Liu, X.-W. 2005, Astron. Nachr., 326, 909 Gurdemir, L., Redfield, S., \& Cuntz, M. 2012, PASA, 29, 141

Hellier, C., Anderson, D. R., Collier Cameron, A., et al. 2009, Nature, 460, 1098 Henry, T. J., Soderblom, D. R., Donahue, R. A., \& Baliunas, S. L. 1996, AJ, 111,439

Kashyap, V. L., Drake, J. J., \& Saar, S. H. 2008, ApJ, 687, 1339

Kraft, R. P., Burrows, D. N., \& Nousek, J. A. 1991, ApJ, 374, 344

Krejčová, T., \& Budaj, J. 2012, A\&A, 540, A82

Lanza, A. F. 2008, A\&A, 487, 1163

Lanza, A. F. 2009, A\&A, 505, 339

Lanza, A. F., Bonomo, A. S., Pagano, I., et al. 2011, A\&A, 525, A14

Lenz, L. F., Reiners, A., \& Kürster, M. 2011, in ASP Conf. Ser. 448, 16th Cambridge Workshop on Cool Stars, Stellar Systems, and the Sun, ed.

C. Johns-Krull (San Francisco, CA: ASP), 1173

Mamajek, E. E., \& Hillenbrand, L. A. 2008, ApJ, 687, 1264 
Moretti, A., Campana, S., Mineo, T., et al. 2005, Proc. SPIE, 5898, 360 Nymeyer, S., Harrington, J., Hardy, R. A., et al. 2011, ApJ, 742, 35

Pevtsov, A. A., Fisher, G. H., Acton, L. W., et al. 2003, ApJ, 598, 1387

Pillitteri, I., Günther, H. M., Wolk, S. J., Kashyap, V. L., \& Cohen, O. 2011, ApJ, 741, L18

Pillitteri, I., Wolk, S. J., Cohen, O., et al. 2010, ApJ, 722, 1216

Poppenhaeger, K., Günther, H. M., \& Schmitt, J. H. M. M. 2012, Astron. Nachr., 333,26

Poppenhäger, K., Lenz, L. F., Reiners, A., Schmitt, J. H. M. M., \& Shkolnik, E. 2011, A\&A, 528, A58

Poppenhäger, K., Robrade, J., \& Schmitt, J. H. M. M. 2010, A\&A, 515, A98

Poppenhäger, K., Robrade, J., Schmitt, J. H. M. M., \& Hall, J. C. 2009, A\&A, 508, 1417

Poppenhäger, K., \& Schmitt, J. H. M. M. 2011, ApJ, 735, 59

Preibisch, T., \& Feigelson, E. D. 2005, ApJS, 160, 390

Rubenstein, E. P., \& Schaefer, B. E. 2000, ApJ, 529, 1031

Saar, S. H., Cuntz, M., Kashyap, V. L., \& Hall, J. C. 2008, in IAU Symp. 249, Exoplanets: Detection, Formation and Dynamics, ed. Y.-S. Sun, S. Ferraz-Mello, \& J.-L. Zhou (Cambridge: Cambridge Univ. Press), 79
Sánchez-Lavega, A. 2004, ApJ, 609, L87

Scharf, C. A. 2010, ApJ, 722, 1547

Schmitt, J. H. M. M. 1997, A\&A, 318, 215

Schmitt, J. H. M. M., \& Liefke, C. 2004, A\&A, 417, 651

Schröter, S., Czesla, S., Wolter, U., et al. 2011, A\&A, 532, A3

Shkolnik, E., Bohlender, D. A., Walker, G. A. H., \& Collier Cameron, A. 2008, ApJ, 676, 628

Shkolnik, E., Walker, G. A. H., Bohlender, D. A., Gu, P.-G., \& Kürster, M. 2005, ApJ, 622, 1075

Southworth, J., Hinse, T. C., Dominik, M., et al. 2009, ApJ, 707, 167

Stelzer, B., \& Neuhäuser, R. 2001, A\&A, 377, 538

Stevens, I. R. 2005, MNRAS, 356, 1053

Triaud, A. H. M. J., Collier Cameron, A., Queloz, D., et al. 2010, A\&A, 524, A25

Walker, G. A. H., Croll, B., Matthews, J. M., et al. 2008, A\&A, 482, 691

Winn, J. N., Fabrycky, D., Albrecht, S., \& Johnson, J. A. 2010, ApJ, 718, L145

Wright, J. T., Fakhouri, O., Marcy, G. W., et al. 2011, PASP, 123, 412

Wright, J. T., Marcy, G. W., Butler, R. P., \& Vogt, S. S. 2004, ApJS, 152, 261 